\title{
Macroscopic Findings Vendor Name
}

National Cancer Institute

\section{Source}

National Cancer Institute. Macroscopic Findings Vendor Name. NCI Thesaurus. Code C119862.

The literal identifier of the vendor or laboratory that performs a macroscopic findings assessment. 